\title{
The Price of (Perceived) Affordance: Commentary for Huron and Berec
}

\author{
ROBERT GJERDINGEN \\ Bienen School of Music, Northwestern University, Illinois
}

\begin{abstract}
It is argued that the symbolic objects in music and musical scores can permit affordances much as physical objects can. This construction of "affordance" places greater emphasis on cultural forms and human memory than the original idea proposed by James J. Gibson, and it aligns itself more closely with the refinements to "affordance" suggested by Donald Norman. For symbolic objects to permit strongly perceived affordances, it may be necessary for perceivers to have developed schematized perception in the course of over-learning culturally significant forms.
\end{abstract}

Submitted 2009 September 24; accepted 2009 October 1.

KEYWORDS: idiomaticism, performance, difficulty, modeling, Gebrauchmuzik

THE article by Huron and Berec (2009) caught my attention both for its originality and its use of the concept of affordance. I have alluded to the same concept in attempts to explain how Italian or Italiantrained musicians of the eighteenth-century were able to improvise, at the keyboard, complete compositions from unfigured basses. My task was to suggest a framework for how even young musicians in the time of Bach could do things that most keyboard players today find quite difficult (or impossible).

The unfigured basses (partimenti) of Francesco Durante, one of the greatest masters of the Italian tradition, often presume contrapuntal imitation in an (unwritten) upper part (think of Bach two-part inventions). Thus the realization of a partimento entailed more than merely guessing chord progressions:

Presuming that the maestro did not immediately tell the student where to place imitations, the student needed to recognize what today we might call the contrapuntal affordances or what an eighteenth-century musician might think of as "opportunities." That is, one needed to learn to recognize the cues and special configurations in the bass that would permit a known contrapuntal treatment. (Gjerdingen, 2007, p. 475)

My mention of "opportunities" was an echo of advice given to young improvisational comedians in the early seventeenth century:

In speaking of commedia dell'arte, Pietro Maria Cecchini (1563-ca. 1630) emphasized that "the actor must see to it that his mind controls his memory (which dispenses the treasure of memorized phrases over the vast field of opportunities constantly offered by comedy)." A young musician with a mind trained to control a "treasure of memorized phrases," some of them learned through singing and playing solfeggi, could quickly apply them to the "opportunities" in a partimento. (2007, p. 465)

By whatever label—affordance or opportunity — "the idea is that an object provides latent action possibilities and that the perception of an object may entail the apprehension of certain functions. A bird may recognize that a particular arrangement of branches provides a good opportunity for nest-building" (Huron \& Berec, 2009, p. 104). I am in complete agreement with the authors on these points.

Of course much depends on what one means by "an object." A bird recognizing "a particular arrangement" of branches may seem analogous to a harpsichord player noticing "cues and special configurations" in a partimento bass or to an organist sensing a match between a memorized pattern and "the local topography" of an unfigured bass (Gjerdingen, 2007). Yet the analogy requires a move from physical objects to symbolic ones. Affordance, as originally envisioned by James J. Gibson (1977), did not 
include a large role for prior learning and culture in the interactions betweens humans and objects. He did allow the possibility of social affordances, but even that idea has had its critics (Harvey, 1981). Were we to limit ourselves to the physical, however, then the story of trumpet affordances might be quite short, as in "the buttons are for pushing, and the mouthpiece is for blowing."

The distinction between the physical and the symbolic begins to blur if we consider virtual objects on a computer screen, things like buttons and sliders. Our knowledge from the physical world transfers to our interactions with these symbolic analogues, and so we push the buttons and drag the sliders. Many metaphors of music - up, down, scale (alluding to "steps" or "stairs") — are similarly analogs of the physical world (Eitan \& Granot, 2006), suggesting that the symbolic world of music may also have affordances.

In Gibson's formulation, one speaks of physical objects "having" affordances, much as if one were talking about physical characteristics like shape or texture. When, however, objects are symbolic and culturally determined, it may be preferable to say that they "permit" affordances. This was a refinement made by Donald Norman (1988), coincidentally now a colleague of mine at Northwestern. In this latter formulation, "perceived affordance" describes an important factor in the interactions between culturally situated humans and the culturally determined objects that they encounter in their environments. For a musician, a musical score is as much part of the environment as the forest is for a hunter or the sea for a sailor.

If we allow objects in the environment to include symbols, then the roles of learning and culture loom quite large. Take, for example, the letters on an automobile license plate that I saw last week while stuck in Chicago traffic: "BE CN U." A number of learned factors-the abbreviated orthography of texting, knowledge of the kinds of messages encoded on license plates, the phonological shape of common phrases as contracted in fluent speech-combined to suggest the stock farewell "(I'll) be seeing you." One could say that the letters on the license plate afforded the farewell, but clearly this is not as straightforward an example as "buttons are for pushing." Similarly the affordance of a phrase-length configuration of notes on a score depends crucially on the background of the performer. Imagine, if you will, two different phrases. In the first, the notes were derived from a random series of pitches. In the second, the notes represent a pattern from a trumpet method book. Though both sets of notes might lie in the same register at the same dynamic marking, in the first case a performer would be forced to engage with a complex and unrecognized pattern of intervals, while in the second case the performer could just "plug in" a single over-learned pattern. The differing degrees of difficulty or idiomaticity of the two patterns would depend at least as much on the musical upbringing of the player as on particular fingerings or registers. In the extreme case where we know nothing of what to do with an object, the object might be said to permit no affordances for us, though it might permit affordances for others.

The price of clearly perceived affordance may thus be a certain degree of schematism derived from the over-learning of culturally significant forms. Like musical forms, cultural forms may exhibit a type of hierarchical structure. For example, the idiomaticity of the nineteenth- and early twentieth-century cornet solos of Herbert L. Clarke (a soloist with the famous band of John Philip Sousa) must surely be grounded in the affordances of cornets and cornetists, given the groundbreaking virtuosity of these works. But a century later, newer scores played by trumpeters trained on these old showpieces may exhibit "second order" affordances. Passages in a more modern score that mimic the contours and dynamic profiles of passages in the old cornet repertory may accrue affordances as a result, and may have a mental idiomaticity that need not be identical to a physical idiomaticity determined from the localized difficulty of playing single intervals. The learned forms of the cornet repertory create Gestalts that can re-emerge as objects with affordances in later repertories. The concept of affordance may thus go well beyond stimulusresponse compatibility to describe a cognitive resonance between an object in the environment and a perceiver's memories of past actions or ideas.

If we admit sonic objects to the class of things permitting affordances, leading to truly "musical" affordances, then listening itself becomes a type of interaction with an aural environment. Several types of listener-oriented music theory could be described as frameworks for recognizing and evaluating affordances. The implication-realization model of Leonard B. Meyer (1973) and Eugene Narmour (1990) would be an obvious case, and, with respect to larger objects, the formal-functions work of William Caplin (1998). Similarly, my own work (2007) with the stock phrases of eighteenth-century music could be described as creating an inventory of historically perceived affordances. In experimental psychology, the many studies on expectancy in music could be classified as exploring affordances, and certainly studies of cue validity such as those by Irene Deliège (1997) explore an area of affordance. My point here is not to list 
every possible connection, but merely to note that affordance is a fertile concept. Like several other aspects of Gibson's innovative approach to psychology, thirty years after his death "affordance" seems more relevant than ever.

\section{REFERENCES}

Caplin, W. (1998). Classical form: A theory of formal functions for the instrumental music of Haydn, Mozart, and Beethoven. New York: Oxford University Press.

Deliège, I \& Mélen, M. (1997). Cue abstraction in the representation of musical form. In Perception and Cognition of Music, ed. Irène Deliège and John Sloboda, pp. 387-412. Hove, UK: Psychology Press.

Eitan, Z. \& Granot, R. (2006). How music moves: Musical parameters and images of motion. Music Perception 23, 221-47.

Gjerdingen, R. O. (2007). Music in the Galant Style. New York: Oxford University Press.

Harvey, J. H. (1981). Cognition, social behavior, and the environment. Hillsdale, NJ: L. Erlbaum.

Huron, D. \& Berec, J. (2009). Characterizing idiomatic organization in music: A theory and case study of musical affordances. Empirical Musicology Review, 4(3): 103-122.

Meyer, L. B. (1973). Explaining music: Essays and explorations. Berkeley: University of California Press.

Narmour, E. (1990). The analysis and cognition of basic melodic structures: the implication-realization model. Chicago: University of Chicago Press. 